\title{
Immune Activation and Inflammatory Biomarkers as Predictors of Venous Thromboembolism in Lymphoma Patients
}

\section{Vladimir Otasevic}

University Clinical Center of Serbia

Biljana Mihaljevic

University Clinical Center of Serbia

Natasa Milic

University of Belgrade

Dejana Stanisavljevic

University of Belgrade

Vojin Vukovic

University Clinical Center of Serbia

Kristina Tomic

University Clinical Center of Serbia

Jawed Fareed

Loyola University Medical Center

Darko Antic ( $\nabla$ darko.antic1510976@gmail.com )

University Clinical Center of Serbia

\section{Research Article}

Keywords: Inflammation, Lymphoma, Risk factor, Venous thromboembolism

Posted Date: November 1st, 2021

DOI: https://doi.org/10.21203/rs.3.rs-1025522/v1

License: (1) (1) This work is licensed under a Creative Commons Attribution 4.0 International License.

Read Full License

Version of Record: A version of this preprint was published at Thrombosis Journal on April 19th, 2022. See the published version at https://doi.org/10.1186/s12959-022-00381-3. 


\section{Abstract}

Background: Lymphomas are characterized by inflammatory soluble mediators that can trigger the development of venous thromboembolism (VTE). However, data on the relationship between specific immune dysregulation and VTE occurrence in lymphoma are scarce. The study aimed to assess the association between inflammatory markers and the risk of VTE development in lymphoma patients.

Methods: The erythrocyte sedimentation rate (ESR), C-reactive protein (CRP), neutrophil-to-lymphocyte ratio (NLR), platelet-to-lymphocyte ratio (PLR), lactate dehydrogenase (LDH), total protein (TP), and albumin were assessed in 706 patients with newly diagnosed or relapsed lymphoma. Data were collected for all VTE events, while the diagnosis of VTE was set objectively based on radiographic studies. ROC (receiver operating characteristic) curve analysis was used to define the optimal cutoff values for predicting VTE.

Results: The majority of patients were diagnosed with aggressive non-Hodgkin lymphoma (58.8\%) and had advanced disease (59.9\%). Sixty-nine patients (9.8\%) developed VTE. The NLR, PLR, ESR, CRP, and LDH were significantly higher in lymphoma patients with VTE, whereas the TP and albumin were significantly lower. In the univariate regression analysis, the NLR, PLR, TP, albumin, LDH, and CRP were prognostic factors for VTE development. In the multivariate regression model, the NLR and CRP were independent prognostic factors for VTE development. ROC curve analysis demonstrated acceptable specificity and sensitivity of the NLR, PLR, and CRP for predicting VTE.

Conclusion: Inflammatory dysregulation plays an important role in VTE development in patients with lymphoma. Widely accessible, simple inflammatory parameters can classify lymphoma patients at risk of VTE development.

\section{Introduction}

Venous thromboembolism (VTE) is a leading cause of cancer-associated thrombosis (CAT) in patients with malignancy. The pathophysiological relationship between VTE development and malignancy was established decades ago.(1) Patients with cancer have up to 7-times higher risk of VTE development than healthy individuals,(2) and VTE is the second leading cause of mortality in cancer patients immediately after cancer progression.(3) Additionally, VTE prolongs the duration of hospitalization and consequently raises the costs of treatment.(4) Further, Khorana et al.(3) identified CAT as the leading cause of mortality in ambulatory patients with active malignancy receiving chemotherapy. The clinical consequences and financial burden of VTE in specific groups of cancer patients have been gaining increased attention.(5)

Lymphomas represent heterogenous group of clonal hematological neoplasms which are characterized by varying clinical course, from utterly indolent to extremely aggressive.(6) Their biological diversity is reflected in various mechanisms through which the disease advances and causes complications. Immune dysregulation, represented by the activation of inflammation, is a particular pathophysiological feature of lymphomas. Inflammation is a result of the complex interaction 
between malignant lymphoma cells and the microenvironment. The level and nature of inflammation dysregulation vary between different types of lymphomas.(7) In patients with a compromised immune system due to both malignancy (primary disease) and cancer treatment, the possibility of infections is substantial, which can generate circumstances for VTE complications.(8) Although the pathways that trigger inflammation are subject to fine modulation and differ based on the type of lymphoma, inflammation has been classically described through white blood cell (WBC) count and standardized acute phase reactants, including $\mathrm{C}$-reactive protein (CRP), sedimentation rate, and fibrinogen level. However, the neutrophil-to-lymphocyte ratio (NLR) and platelet-to-lymphocyte ratio (PLR) are newer biomarkers of systemic inflammation that are better inflammatory indicators than WBC. The NLR and PLR have been widely studied in different medical fields. Additionally, their potential strength for predicting VTE in cancer patients receiving chemotherapy has been emphasized in several recent publications. $(9,10)$ However, their predictive performance and reliability have not been studied in patients with lymphoma.

Inflammation and inflammation-related conditions are associated with an increased risk of VTE because of the dysregulation of multiple pathophysiological pathways, including venous stasis, hypercoagulability, inflammation, interleukin- 6 expression, and inhibition of natural inhibitors of coagulation and anticoagulants.(11) The inflammatory and thrombotic pathways overlap.(11) Immunothrombosis, a relatively new term coined by Engelmann and Massberg,(12) emphasizes the role of innate immunity in VTE development. However, data on the relationship between specific immune dysregulation in lymphoma and VTE occurrence are scarce. Therefore, the aim of our study was to assess the association between inflammatory markers and the risk of VTE development in lymphoma patients receiving chemotherapy and the relationship between VTE and the treatment course of patients with lymphoma.

\section{Methods}

\section{Study population}

The study included 706 patients with newly diagnosed or relapsed lymphoma (including non-Hodgkin lymphoma [NHL] and Hodgkin lymphoma [HL] and excluding chronic lymphocytic leukemia [CLL] and the leukemic phases of all lymphomas) at the Clinic for Hematology, University Clinical Center of Serbia (UCCS). The study protocol has been approved by the UCCS's Ethics Committee, while the informed consent was obtained from all the participants. The time frame from which eligible patients were selected was January 2010 to November 2019. Patients with CLL and other leukemic phases of lymphomas were excluded because their differential WBC count is shifted and affects the NLR and PLR, rendering these values invalid. Data for patients with newly diagnosed and relapsed lymphoma were collected for all VTE events. VTE was diagnosed objectively based on radiographic studies, including compression ultrasonography, contrast-enhanced thoracic computed tomography, and magnetic resonance imaging for central nervous system (CNS) thrombosis, as well as clinical examination and laboratory evaluation. 
All probable cases of VTE were reviewed by a final diagnosis committee composed of two specialists (an internist and a radiologist).

\section{Laboratory investigations}

Blood samples from lymphoma patients included in the study were collected using vacuum tubes. Samples were anticoagulated with EDTA, and machine-automatized complete blood count (CBC) with leukocyte differential counts were obtained. The NLR and PLR were calculated using the CBC with differential counts. Citrated blood samples were analyzed in batches using commercially available ELISA kits at the University Clinical Center of Serbia, and the following biochemical parameters were obtained: erythrocyte sedimentation rate (ESR), C-reactive protein (CRP), lactate dehydrogenase (LDH), fibrinogen, total protein (TP), and albumin.

\section{Statistical analysis}

Categorical variables are displayed as counts with percentages, and numerical variables are presented as medians with ranges. Normality of distribution was assessed using the Kolmogorov-Smirnov test. LDH, TP, and albumin were transformed to dichotomous categorical variables and defined as "under the lower reference range limit," "in the reference range," or "over the upper reference range limit." Differences between lymphoma patients who developed thrombosis and those without thrombosis were assessed using the Mann-Whitney test for numerical variables and the chi-square test for categorical variables. ROC (receiver operating characteristic) curve analysis was used to define the best cutoff values for predicting VTE. Multivariate logistic regression analysis was used to identify significant predictors of thrombosis in patients with lymphoma. Significant variables from the univariate logistic regression analysis were entered into the multivariate analysis. The results are presented as odds ratios (ORs) with corresponding $95 \%$ confidence intervals (Cls). Statistical significance was set at $p<0.05$. Statistical analysis was performed using IBM SPSS statistical software (SPSS for Windows, release 25.0, SPSS, Chicago, IL, USA).

\section{Results}

The mean age of the patients included in the study was 52.8 years (range, $18-89$ years); $53 \%$ of the patients were men. A total of 415 patients $(58.8 \%)$ had aggressive NHL, $172(24.3 \%)$ had indolent $\mathrm{NHL}$, and 119 (16.9\%) had HL. Most of the patients were newly diagnosed (90.4\%) and had advanced disease, with Ann Arbor stages III and IV accounting for $20.6 \%$ and $39.3 \%$ of cases, respectively. Most patients had good performance status (Eastern Cooperative Oncology Group Performance Status [ECOG PS] 0-1: $81.7 \%$;), and B symptoms were present in $55.8 \%$ of patients. A "bulky" tumor mass was observed in $30.7 \%$ of patients, and mediastinal involvement was present in $31.4 \%$ of patients. 
Median follow up was 25 months. Sixty-nine patients (9.8\%) developed VTE events: 39 developed deep vein thrombosis (DVT) of the extremities, 3 developed abdominal vein thrombosis, 12 developed superficial vein thrombosis, 11 developed jugular vein thrombosis, and 16 developed pulmonary embolisms (some patients had more than one thrombotic event). The majority of patients developed VTE during treatment $(52.1 \%)$, but $46.5 \%$ of patients were diagnosed with VTE prior to treatment initiation, and $1.2 \%$ developed VTE after completion of treatment. VTE was more frequent in patients with aggressive lymphoma (11.8\%) than in patients with $\mathrm{HL}(8.4 \%)$ and indolent lymphoma (5.8\%). Most lymphoma patients with VTE had advanced disease (stage III, 29\%; stage IV, 34.8\%).

The demographic and clinical characteristics of lymphoma patients with and without VTE are presented in Table 1. Compared to patients without VTE, the NLR, PLR, ESR, CRP, and LDH were significantly higher in lymphoma patients with VTE $(p=0.001, p=0.001, p=0.023, p<0.001$, and $p=0.035$, respectively), whereas the TP and albumin were significantly lower $(p=0.024$ and $p=0.032$, respectively).

Table 1

Demographic and clinical characteristics of lymphoma patients with and without VTE 


\begin{tabular}{|c|c|c|c|}
\hline Characteristic & $\begin{array}{l}\text { Lymphoma patients } \\
\text { without VTE }\end{array}$ & $\begin{array}{l}\text { Lymphoma patients with } \\
\text { VTE }\end{array}$ & $\mathrm{p}$ \\
\hline Age, median (range) & $56(18-87)$ & $54(19-89)$ & 0.898 \\
\hline Men/women & $337 / 300$ & $37 / 32$ & 0.900 \\
\hline $\begin{array}{l}\text { Hemoglobin, } \mathrm{g} / \mathrm{L} \text {, median } \\
\text { (range) }\end{array}$ & $124(51-172)$ & $117(87-141)$ & 0.017 \\
\hline $\begin{array}{l}\text { Leukocytes, } x 10^{9} / \mathrm{L} \text {, median } \\
\text { (range) }\end{array}$ & $7.4(0.4-28.5)$ & $8.5(4.6-16.6)$ & 0.132 \\
\hline $\begin{array}{l}\text { Platelets, } \times 10^{9} / \mathrm{L} \text {, median } \\
\text { (range) }\end{array}$ & $248(29-613)$ & $283(103-678)$ & 0.034 \\
\hline NLR, median (range) & $2.7(0.2-32.5)$ & $3.79(0.7-160.5)$ & 0.001 \\
\hline PLR, median (range) & $10.1(0.3-193.3)$ & $14.5(483.3)$ & 0.001 \\
\hline ESR, mm/h, median (range) & $26(2-150)$ & $38(2-150)$ & 0.023 \\
\hline CRP, mg/L, median (range) & $9.9(0.1-274.6)$ & $30.6(0.8-251.8)$ & $<0.001$ \\
\hline $\begin{array}{l}\text { Fibrinogen, mg/L, median } \\
\text { (range) }\end{array}$ & $5.3(1-13.2)$ & $5.7(1.9-11.8)$ & 0.351 \\
\hline $\begin{array}{l}\mathrm{LDH}>\text { reference range limit, } n \\
(\%)\end{array}$ & $328(51.5)$ & $45(65.1)$ & 0.035 \\
\hline $\mathrm{TP}<$ reference range limit, $\mathrm{n}(\%)$ & $258(40.5)$ & $39(56.6)$ & 0.024 \\
\hline $\begin{array}{l}\text { Albumin < reference range limit, } \\
\mathrm{n}(\%)\end{array}$ & $70(11)$ & $15(21.2)$ & 0.032 \\
\hline B symptomatology, n (\%) & $360(56.6)$ & $52(75.4)$ & 0.001 \\
\hline "Bulky" tumor mass, n (\%) & $182(28.5)$ & $34(49.3)$ & $<0.001$ \\
\hline Extranodal localization, n (\%) & $368(57.8)$ & $35(50.7)$ & 0.259 \\
\hline Mediastinal involvement, $\mathrm{n}(\%)$ & $189(29.6)$ & $32(46.4)$ & 0.004 \\
\hline
\end{tabular}

In the univariate regression analysis, the NLR, PLR, TP, albumin, LDH, and CRP were found to be prognostic factors for VTE development in lymphoma patients (Table 2). In the subgroup analysis of superficial vein thrombosis events exclusion, both NLR and PLR remained prognostic factors for VTE development. B-symptomatology, a "bulky" tumor mass, mediastinal involvement, and ECOG PS were the significant clinicopathological prognostic factors for VTE development in lymphoma patients $(p=0.001$, $p=0.001, p=0.005$, and $p=0.015$, respectively) (Table 2 ). In the multivariate regression model, the NLR 
and CRP were found to be independent prognostic factors for VTE development in patients with lymphoma $(p=0.049, \mathrm{OR}=1.04,95 \% \mathrm{Cl}: 1-1.08$ and $p=0.02, \mathrm{OR}=1,95 \% \mathrm{Cl}: 1-1.01$, respectively).

Table 2

Univariate regression models of TP, albumin, LDH, CRP, ESR, NLR, PLR, B-symptomatology, "bulky" tumor mass, mediastinal involvement by tumor mass and ECOG PS.

\begin{tabular}{|llll|}
\hline Variable & $\mathrm{p}$ & $\mathrm{OR}$ & $95 \% \mathrm{Cl}$ for OR \\
\hline TP & 0.026 & 1.91 & $1.08-3.39$ \\
\hline Albumin & 0.036 & 2.16 & $1.05-4.46$ \\
\hline LDH & 0.037 & 1.78 & $1.03-3.06$ \\
\hline CRP & 0.008 & 2.75 & $1.29-5.84$ \\
\hline ESR & 0.18 & 1.76 & $0.78-4.02$ \\
\hline NLR & 0.001 & 2.5 & $1.48-4.21$ \\
\hline PLR & 0.003 & 2.24 & $1.31-3.83$ \\
\hline B-symptomatology & 0.001 & 2.64 & $1.49-4.67$ \\
\hline "Bulky" tumor mass & 0.001 & 2.43 & $1.47-4.01$ \\
\hline Mediastinal involvement & 0.005 & 2.05 & $1.24-3.39$ \\
\hline ECOG PS & 0.015 & 1.99 & $1.14-3.5$ \\
\hline $\begin{array}{l}\text { Legend: OR, odds ratio; Cl, confidence interval; TP, total proteins; LDH, lactate dehydrogenase; CRP, C- } \\
\text { reactive protein; ESR, erythrocyte sedimentation rate; NLR, neutrophil to lymphocyte ratio; PLR, platelet } \\
\text { to lymphocyte ratio; ECOG PS, Eastern Oncology Cooperative Group Performance Status. }\end{array}$ \\
\hline
\end{tabular}

The ROC curve analysis demonstrated acceptable specificity and sensitivity of the NLR, PLR, and CRP in predicting VTE ( $p=0.001, p=0.001$, and $p<0.001$, respectively) (Figure $A, B$ and $C$ ). A high NLR was defined as an NLR of 3 or higher, a high PLR as a PLR of 10 or higher, and a high CRP level as CRP $>20$ $\mathrm{mg} / \mathrm{L}$.

There was no difference in the use of thromboprophylaxis between lymphoma patients with and without $\operatorname{VTE}(13 \%$ vs. $18.4 \%, p=0.268)$.

A poor therapeutic response to chemotherapy and immunotherapy was associated with the development of VTE $(p=0.011)$. Complete remission was less frequent in lymphoma patients who developed VTE than in those who did not develop VTE $(36.9 \%$ vs. $53.6 \%, p=0.011)$. Patients receiving intensive first-line or "salvage" chemotherapeutic regimens experienced a higher VTE rate than patients treated with standard first-line therapy regimens, such as R-CHOP, CHOP, and ABVD $(18.2 \%$ vs $7.3 \%, p<0.001)$. 


\section{Discussion}

In the study we aimed to evaluate the connection between inflammatory markers and the risk of VTE development in the cohort of lymphoma patients, and the relationship between VTE and the treatment course of patients with lymphoma. Our analysis found that the inflammatory markers correlate well with the risk for VTE development in lymphoma patients, with NLR and CRP being the most accurate VTE predictive markers, based on statistical analysis. Further, we identified that an insufficient therapeutic response on (immuno)chemotherapy is a risk factor for VTE in lymphoma patients. Summarizing, immune dysregulation in lymphoma settings has a substantial impact on VTE occurrence.

In our study of patients with different types of lymphoma, the rate of VTE development was $9.8 \%$. In a meta-analysis by Caruso et al.,(13) which included 18,018 patients with lymphoma, the rate of VTE development was $6.4 \%$. In that study, a higher rate of VTE development was observed in NHL patients than in HL patients. In a study by Mahajan et al.,(14) the cumulative 2-year incidences of acute VTE were $2.1 \%, 4.8 \%$, and $4.5 \%$ in patients with low-grade, intermediate/aggressive, and high-grade lymphomas, respectively. Two studies $(15,16)$ focusing only on diffuse large B-cell lymphoma (DLBCL) found that the rate of VTE development was $11 \%$ and $11.1 \%$, respectively. In a study examining the frequency of VTE in cancer patients, Khorana et al.(17) observed that $4.8 \%$ of NHL patients developed VTE, whereas $4.6 \%$ of HL patients developed VTE. In the study by Antic et al.,(18) the rates of VTE development among lymphoma patients were $5.3 \%$ in the derivation cohort and $5.8 \%$ in the validation cohort. In a recently published article(19) focusing on DLBCL and follicular lymphoma, the reported rate of VTE development was $13.4 \%$. These observed variations in the VTE rate in lymphoma patients are notable and may have several causes, including focusing on distinctive types of lymphoma, study methodology (e.g., retrospective vs. prospective), and publication time (more recent studies have been dedicated to CAT). Our results are similar to those of studies focusing only on aggressive lymphoma, which is in accordance with the fact that more than half of our study population had aggressive lymphoma.

In our study, the NLR, PLR, ESR, CRP, and LDH were significantly higher in lymphoma patients with VTE than in those without VTE, whereas the TP and albumin were significantly lower in lymphoma patients with VTE than in those without VTE. The ROC curve analysis indicated acceptable specificity and sensitivity of the NLR, PLR, and CRP in predicting VTE in lymphoma patients. In particular, the univariate regression analysis indicated that the NLR, PLR, TP, albumin, LDH, and CRP were prognostic factors for VTE development in lymphoma patients, although the multivariate regression model demonstrated that only the NLR and CRP were independent prognostic factors for VTE development. Both the NLR and PLR have been used as prognostic markers in a variety of pathological conditions, including sepsis, lupus erythematosus and solid tumors.(20) Additionally, the NLR and PLR have been suggested as adverse prognostic markers in patients with DLBCL(21) and mantle cell lymphoma,(22) although some publications have found conflicting results.(23) Regarding the association of the NLR and PLR with thrombotic events, some previous studies have shown the predictive power of the NLR and PLR for VTE development.(10,24) In contrast, Artoni et al. $(25)$ failed to find an association of the NLR and PLR with an 
increased risk of VTE or cerebral vein thrombosis. To the best of our knowledge, there are no published studies using the NLR and PLR to assess the risk of VTE in lymphoma patients.

An increasing number of studies aim to define the relationship between inflammation and thrombosis, as well as the specific mechanisms underlying this relationship, but the most important mechanisms are yet to be discovered. The best studied mechanisms that have been shown to trigger thrombosis development or have been frequently observed in patients who develop thrombosis are increased levels of tumor necrosis factor-alpha (TNF-a),(26) hyperexpression of interleukin-6,(11) neutrophil extracellular traps, (27) soluble CD40 ligand,(28) and microparticles (MPs).(29) Kapoor et al.(30) significantly advanced our understanding of these processes by introducing a fourth element to Virchow's triad-immune dysregulation, naming it the "tetrad of thrombosis." They clearly stressed that there is a sufficient amount of evidence supporting the impact of immune dysregulation on the pathophysiology of thrombosis. A few publications identified higher CRP in patients with VTE (mainly DVT),(31) whereas the study by Antic et al.(32) published results similar to ours, showing the effect of a broad inflammatory and hemostatic biomarker spectrum (including D-dimer, Factor XIIla, von Willebrand factor, TNF-a, protein S, $\beta 2$ Glycoprotein I, MPs, urokinase-like plasminogen activator, fibronectin, and plasminogen activator inhibitor type 1).

Similar to the results of previous studies, $(13,14,33)$ we found that patients with advanced disease more frequently developed VTE, although this was not statistically significant. A "bulky" tumor mass, mediastinal involvement, and ECOG PS were identified as prognostic factors for VTE development in lymphoma patients in the univariate analysis. A large mediastinal tumor mass is an important risk factor for the development of VTE, mainly because of the mechanical compression of blood vessels and consequent narrowing of the lumen.(34,35) Performance status is included in newer VTE risk assessment models, underlining its importance in VTE development.(18,35) Immobility has been recognized as a contributing factor for VTE. It is of particular important in patients with CNS lymphoma, as they have a strikingly high rate of VTE development (59.5\%).(36)

In our cohort, patients with aggressive lymphoma had a higher rate of VTE development (11.8\%) than did patients with indolent lymphoma (5.8\%) and $\mathrm{HL}(8.4 \%)$. Aggressive histology is predisposed to complicate the clinical course of lymphoma due to VTE. $(13,14,19,33,35,37)$ However, one large study by Sanfilippo et al.(38) concluded that the VTE risk for DLBCL is lowered after adjusting for additional risk factors. In general, aggressive lymphomas have higher proliferation rate which enables them to advance promptly and to obtain VTE risk factors more rapidly ("bulky" tumor mass, extranodal localizations, poor performance status), consequently increasing the risk for VTE development.

Complementary to our results, the predominant timing of VTE occurrence in lymphoma patients is prior to or within 3 months from the initiation of specific hematologic treatment. $(34,39,40)$ These data draw attention to the role of thromboprophylaxis, which remains underused in cancer patients. $(18,41)$ Considering the absence of statistical significance for thromboprophylaxis between lymphoma patients with and without VTE, our data confirm the underutilization of thromboprophylaxis. There are 
several reasons why thromboprophylaxis continues to be underused in lymphoma patients: the lack of reliable and widely accepted usage of a VTE risk assessment model for this heterogeneous group of patients, the lack of prospective studies with risk stratification and randomization for thromboprophylaxis,(37) excessively diverse data throughout the literature concerning this topic, and overestimation of bleeding risk in combination with anticoagulant therapy in cancer patients by clinicians. Further disease specific and adequately designed clinical trials on thromboprophylaxis are required in order to achieve high quality evidence to ameliorate clinical guidelines.

Importantly, lymphoma patients who achieved unsatisfactory therapeutic responses were more susceptible to the development of VTE. This finding is in accordance with published data that confirm the connection between aggressive lymphoma and advanced stage, resulting in shorter overall survival (OS) and a higher mortality rate. $(14,33,34,42)$ However, one study(43) did not observe an OS difference between lymphoma patients with and without VTE. The biology of aggressive lymphoma leads to aggravate clinical course. Moreover, immune dysregulation in aggressive lymphoma subtypes is probably impaired to a greater extent, which contributes to the risk for VTE occurrence.

In our study, patients receiving intensive first-line or "salvage" chemotherapeutic regimens experienced a higher rate of VTE development than patients treated with standard first-line therapy regimens (R-CHOP, $\mathrm{CHOP}$, and ABVD). Chemotherapy itself is known to be a risk factor for VTE development.(3,35) The incidence of VTE is higher in lymphoma patients treated with dose-intense regimens.(44) Further, anthracycline drugs are associated with an increased risk of VTE. $(19,38)$ Intensive first-line therapeutic regimens are used to treat more aggressive lymphoma subtypes, and both intensive regimens and aggressive subtypes are potential risk factors for VTE development. Relapsing lymphomas are inclined to follow a more aggressive clinical course, primarily because of the biology of the disease and the development of resistant features. Consequently, those patients are treated with more intensive, so-called "salvage," chemotherapeutic regimens. These patients frequently have other VTE risk factors, such as poor performance status and advanced disease, which significantly increase the risk of VTE development.

Our data comes with several limitations to be mentioned. The main limitation is the heterogeneity of the study population, which possibly affects the results and subsequent conclusions. The impact of VTE onto survival rates of lymphoma patients was out of scope of this study. Perhaps, that would further contribute to the assessment of actual clinical impact of VTE in lymphoma patients.

\section{Conclusion}

In conclusion, immune activation represents a distinctive feature of lymphomas, especially aggressive lymphomas. Dysregulation of inflammation plays an important role in VTE development in patients with lymphoma. The findings of this study demonstrated that easily and widely accessible, simple parameters that reflect the level of inflammation have the ability to identify lymphoma patients at risk for VTE who may be candidates for thromboprophylaxis. In addition, the possible use of anti-inflammatory drugs in 
this specific group of patients would extend the tools for VTE prophylaxis. Further studies are required to better understand VTE in lymphoma settings and the utilization of these inflammatory markers in VTE risk assessment.

\section{Abbreviations}

VTE - venous thromboembolism

CAT - cancer-associated thrombosis

WBC - white blood cell

CRP - C-reactive protein

NLR - neutrophil-to-lymphocyte ratio

PLR - platelet-to-lymphocyte ratio

NHL - non-Hodgkin lymphoma

HL - Hodgkin lymphoma

CLL - chronic lymphocytic leukemia

UCCS - University Clinical Center of Serbia

CNS - central nervous system

CBC - complete blood count

ESR - erythrocyte sedimentation rate

$\mathrm{LDH}$ - lactate dehydrogenase

TP - total protein

ROC - receiver operating characteristic

$\mathrm{OR}$ - odds ratio

$\mathrm{Cl}$ - confidence intervals

ECOG PS - Eastern Cooperative Oncology Group Performance Status

DVT - deep vein thrombosis 
DLBCL - diffuse large B-cell lymphoma

TNF-a - tumor necrosis factor-alpha

MP - microparticles

OS - overall survival

\section{Declarations}

\section{Ethics approval:}

Mentioned in the manuscript.

\section{Competing interests:}

Authors declare no conflict of interest.

\section{Acknowledgments:}

Not applicable.

\section{Consent for publication:}

Not applicable.

\section{Availability of data and materials:}

The datasets used and analysed during the current study are available from the corresponding author on reasonable request.

\section{Funding:}

Not applicable.

\section{Authors' information:}

Not applicable. 


\section{Authors' contributions:}

Vladimir Otasevic - first author of the manuscript

Biljana Mihaljevic - review of the manuscript, helped with introduction and discussion part

Natasa Milic - statistical analysis and methods

Dejana Stanisavljevic - statistical analysis and methods

Vojin Vukovic - discussion reviewer, added few significant ideas

Kristina Tomic - introduction and supplements author

Jawed Fareed - article review, helped with several important improvements to the manuscript

Darko Antic - the leader of the project

All authors reviewed the manuscript.

\section{References}

1. Mukai M, Oka T. Mechanism and management of cancer-associated thrombosis. J Cardiol. 2018;72(2):89-93.

2. Blom JW, Doggen CJM, Osanto S, Rosendaal FR. Malignancies, prothrombotic mutations, and the risk of venous thrombosis. JAMA. 2005;293(6):715-22.

3. Khorana AA, Francis CW, Culakova E, Kuderer NM, Lyman GH. Thromboembolism is a leading cause of death in cancer patients receiving outpatient chemotherapy. Vol. 5, Journal of thrombosis and haemostasis: JTH. England; 2007. p. 632-4.

4. Donnellan E, Khorana AA. Cancer and Venous Thromboembolic Disease: A Review. Oncologist. 2017/02/07. 2017;22(2):199-207.

5. Gussoni G, Foglia E, Frasson S, Casartelli L, Campanini M, Bonfanti M, et al. Real-world economic burden of venous thromboembolism and antithrombotic prophylaxis in medical inpatients. Thromb Res. 2013;131(1):17-23.

6. Armitage JO, Gascoyne RD, Lunning MA, Cavalli F. Non-Hodgkin lymphoma. Lancet (London, England). 2017;390(10091):298-310.

7. Carbone A, Tripodo C, Carlo-Stella C, Santoro A, Gloghini A. The role of inflammation in lymphoma. Adv Exp Med Biol. 2014;816:315-33.

8. Koch A, Meesters MI, Scheller B, Boer C, Zacharowski K. Systemic endotoxin activity correlates with clot formation: an observational study in patients with early systemic inflammation and sepsis. Crit 
Care. 2013;17(5):R198.

9. Wang Q, Ma J, Jiang Z ML. Prognostic value of neutrophil-to-lymphocyte ratio and platelet-tolymphocyte ratio in acute pulmonary embolism: a systematic review and meta-analysis. Int Angiol. 2018;37:4-11.

10. Farah R, Nseir W, Kagansky D, Khamisy-Farah R. The role of neutrophil-lymphocyte ratio, and mean platelet volume in detecting patients with acute venous thromboembolism. J Clin Lab Anal. 2020;34(1):e23010.

11. Vazquez-Garza E, Jerjes-Sanchez C, Navarrete A, Joya-Harrison J, Rodriguez D. Venous thromboembolism: thrombosis, inflammation, and immunothrombosis for clinicians. J Thromb Thrombolysis. 2017;44(3):377-85.

12. Engelmann B, Massberg S. Thrombosis as an intravascular effector of innate immunity. Nat Rev Immunol. 2013;13(1):34-45.

13. Caruso V, Di Castelnuovo A, Meschengieser S, Lazzari MA, de Gaetano G, Storti S, et al. Thrombotic complications in adult patients with lymphoma: a meta-analysis of 29 independent cohorts including 18018 patients and 1149 events. Blood. 2010;115(26):5322-8.

14. Mahajan A, Wun T, Chew H, White RH. Lymphoma and venous thromboembolism: influence on mortality. Thromb Res. 2014;133 Suppl:S23-8.

15. Yokoyama K, Murata M, Ikeda Y, Okamoto S. Incidence and risk factors for developing venous thromboembolism in Japanese with diffuse large b-cell lymphoma. Thromb Res. 2012;130(1):7-11.

16. Borg IH, Bendtsen MD, Bogsted M, Madsen J, Severinsen MT. Incidence of venous thromboembolism in patients with diffuse large B-cell lymphoma. Leuk Lymphoma. 2016;57(12):2771-6.

17. Khorana AA, Francis CW, Culakova E, Kuderer NM, Lyman GH. Frequency, risk factors, and trends for venous thromboembolism among hospitalized cancer patients. Cancer. 2007;110(10):2339-46.

18. Antic D, Milic N, Nikolovski S, Todorovic M, Bila J, Djurdjevic P, et al. Development and validation of multivariable predictive model for thromboembolic events in lymphoma patients. Am J Hematol. 2016;91(10):1014-9.

19. Dharmavaram G, Cao S, Sundaram S, Ayyapan S, Boughan K, Gallogly M, et al. Aggressive Lymphoma Subtype Is A Risk Factor for Venous Thrombosis. Development of Lymphoma - Specific Venous Thrombosis Prediction Models. Am J Hematol. 2020;

20. Wang S, Liu H, Wang Q, Cheng Z, Sun S, Zhang Y, et al. Neutrophil-to-Lymphocyte Ratio and Plateletto-Lymphocyte Ratio Are Effective Predictors of Prognosis in Patients with Acute Mesenteric Arterial Embolism and Thrombosis. Ann Vasc Surg. 2018;49:115-22.

21. Wang S, Ma Y, Sun L, Shi Y, Jiang S, Yu K, et al. Prognostic Significance of Pretreatment Neutrophil/Lymphocyte Ratio and Platelet/Lymphocyte Ratio in Patients with Diffuse Large B-Cell Lymphoma. Biomed Res Int. 2018;2018:9651254.

22. Haydaroglu Sahin H. Can the prognosis of mantle cell lymphoma be predicted by simple CBC counts? Medicine (Baltimore). 2019;98(30):e16180. 
23. Azuma Y, Nakaya A, Fujita S, Satake A, Nakanishi T, Tsubokura Y, et al. Neutrophil-to-lymphocyte ratio (NLR) fails to predict outcome of diffuse large B cell lymphoma. Leuk Res reports. 2019;12:100173.

24. Ferroni P, Riondino S, Formica V, Cereda V, Tosetto L, La Farina F, et al. Venous thromboembolism risk prediction in ambulatory cancer patients: clinical significance of neutrophil/lymphocyte ratio and platelet/lymphocyte ratio. Int J cancer. 2015;136(5):1234-40.

25. Artoni A, Abbattista M, Bucciarelli P, Gianniello F, Scalambrino E, Pappalardo E, et al. Platelet to Lymphocyte Ratio and Neutrophil to Lymphocyte Ratio as Risk Factors for Venous Thrombosis. Clin Appl Thromb Hemost. 2018;24(5):808-14.

26. Saghazadeh A, Rezaei N. Inflammation as a cause of venous thromboembolism. Crit Rev Oncol Hematol. 2016;99:272-285.

27. Fuchs TA, Brill A, Duerschmied D, Schatzberg D, Monestier M, Myers DDJ, et al. Extracellular DNA traps promote thrombosis. Proc Natl Acad Sci U S A. 2010;107(36):15880-5.

28. Ferroni P, Santilli F, Guadagni F, Basili S, Davi G. Contribution of platelet-derived CD40 ligand to inflammation, thrombosis and neoangiogenesis. Curr Med Chem. 2007;14(20):2170-80.

29. Ardoin SP, Shanahan JC, Pisetsky DS. The role of microparticles in inflammation and thrombosis. Scand J Immunol. 2007;66(2-3):159-65.

30. Kapoor S, Opneja A, Nayak L. The role of neutrophils in thrombosis. Thromb Res. 2018/08/09. 2018;170:87-96.

31. Borgel D, Bianchini E, Lasne D, Pascreau T, Saller F. Inflammation in deep vein thrombosis: a therapeutic target? Hematol (United Kingdom). 2019;24(1):742-50.

32. Antic D, Milic N, Bontekoe E, Hoppensteadt D, Djurasinovic V, Vukovic V, et al. Biomarkers of Hemostatic Dysregulation and Inflammation in Lymphoma: Potential Relevance to Thrombogenesis. Blood. 2019;134(Supplement_1):4945.

33. Yokoyama K. Thrombosis in lymphoma patients and in myeloma patients. Keio J Med. 2015;64(3):37-43.

34. Lekovic D, Miljic $P$, Mihaljevic B. Increased risk of venous thromboembolism in patients with primary mediastinal large B-cell lymphoma. Thromb Res. 2010;126(6):477-80.

35. Falanga A, Marchetti M, Russo L. Venous thromboembolism in the hematologic malignancies. Curr Opin Oncol. 2012;24(6):702-10.

36. Goldschmidt N, Linetsky E, Shalom E, Varon D, Siegal T. High incidence of thromboembolism in patients with central nervous system lymphoma. Cancer. 2003;98(6):1239-42.

37. Kekre N, Connors JM. Venous thromboembolism incidence in hematologic malignancies. Blood Rev. 2019;33:24-32.

38. Sanfilippo KM, Wang TF, Gage BF, Luo S, Riedell P, Carson KR. Incidence of venous thromboembolism in patients with non-Hodgkin lymphoma. Thromb Res. 2016;143:86-90.

39. Antic D, Jelicic J, Vukovic V, Nikolovski S, Mihaljevic B. Venous thromboembolic events in lymphoma patients: Actual relationships between epidemiology, mechanisms, clinical profile and treatment. 
Blood Rev. 2018;32(2):144-58.

40. Colombo R, Gallipoli P, Castelli R. Thrombosis and hemostatic abnormalities in hematological malignancies. Clin Lymphoma Myeloma Leuk. 2014;14(6):441-50.

41. Brenner B, Hull R, Arya R, Beyer-Westendorf J, Douketis J, Elalamy I, et al. Evaluation of unmet clinical needs in prophylaxis and treatment of venous thromboembolism in high-risk patient groups: cancer and critically ill. Thromb J. 2019;17(1):6.

42. Byun JM, Hong J, Yoon S-S, Koh Y, Ock C-Y, Kim TM, et al. Incidence and characteristics of venous thromboembolism in Asian patients with primary central nervous system lymphoma undergoing chemotherapy. Thromb Res. 2019;183:131-5.

43. Park LC, Woo S, Kim S, Jeon H, Ko YH, Kim SJ, et al. Incidence, risk factors and clinical features of venous thromboembolism in newly diagnosed lymphoma patients: Results from a prospective cohort study with Asian population. Thromb Res. 2012;130(3):E6-12.

44. Borchmann S, Muller H, Hude I, Fuchs M, Borchmann P, Engert A. Thrombosis as a treatment complication in Hodgkin lymphoma patients: a comprehensive analysis of three prospective randomized German Hodgkin Study Group (GHSG) trials. Ann Oncol Off J Eur Soc Med Oncol. 2019;30(8):1329-34.

\section{Figures}




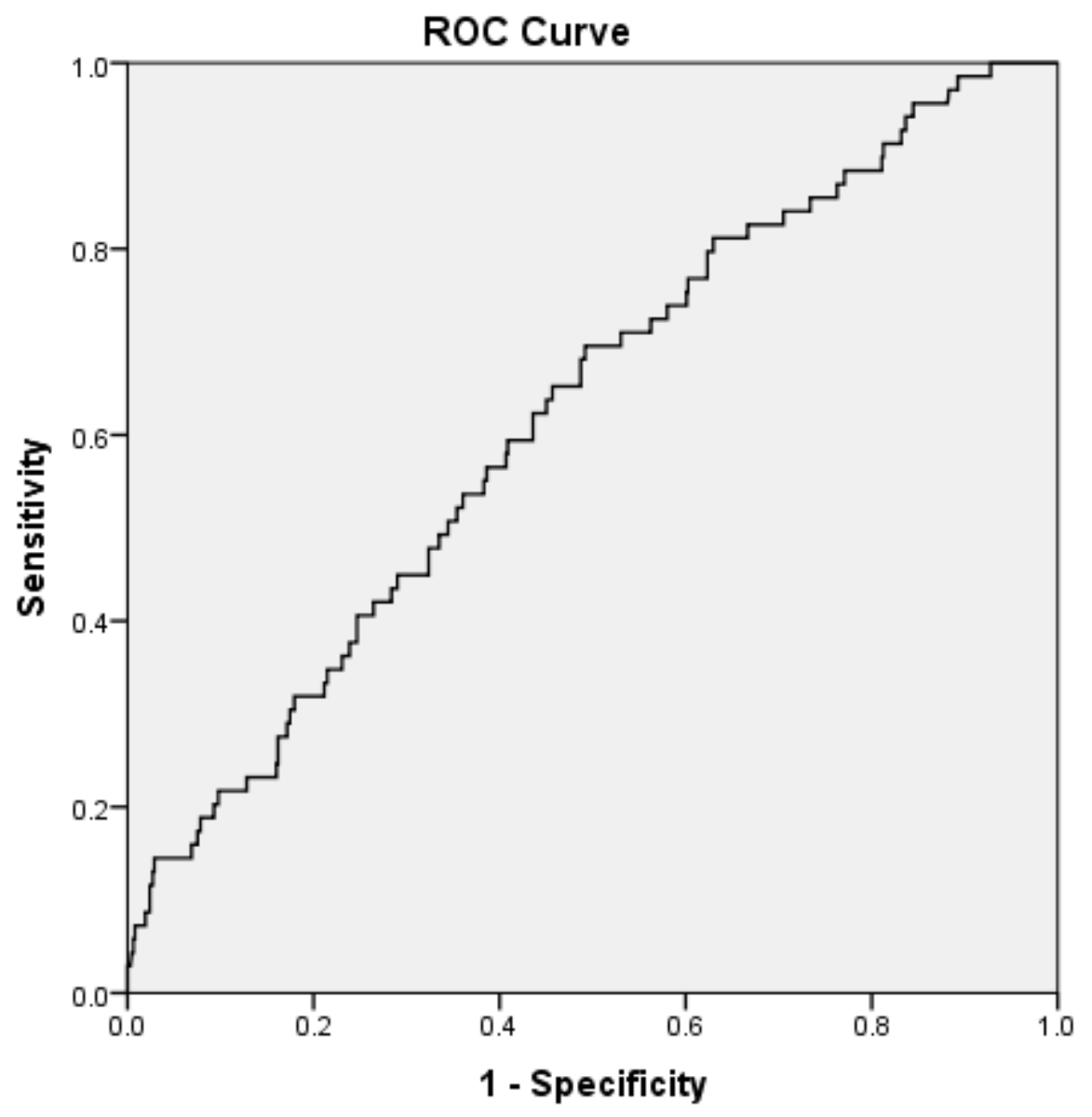

Figure 1

NLR ROC curve Legend: ROC, receiver operating characteristic; NLR, neutrophil to lymphocyte ratio; PLR, platelet to lymphocyte ratio; CRP, C-reactive protein; AUC, area under curve. 


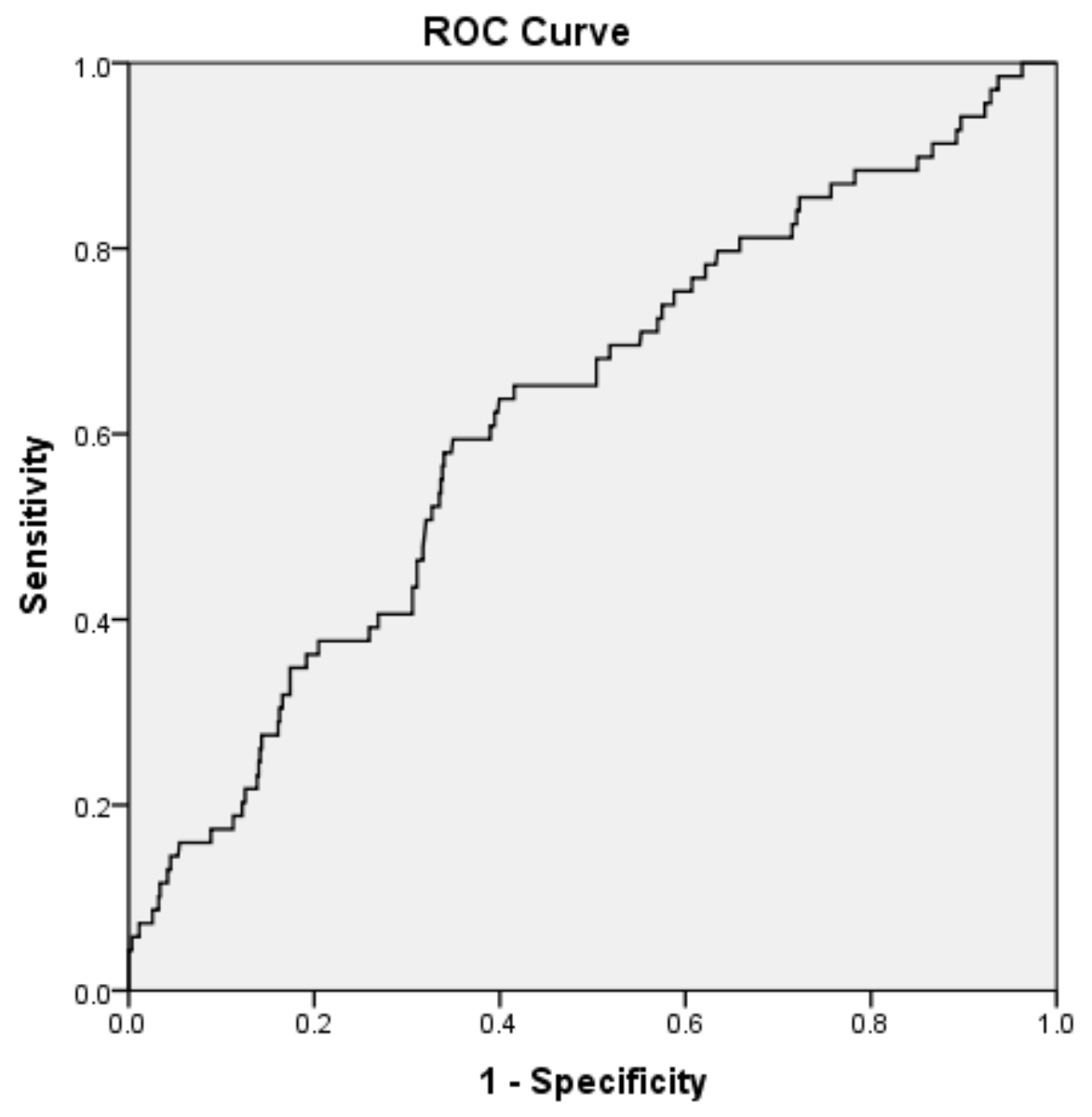

Figure 2

PLR ROC curve Legend: ROC, receiver operating characteristic; NLR, neutrophil to lymphocyte ratio; PLR, platelet to lymphocyte ratio; CRP, C-reactive protein; AUC, area under curve. 


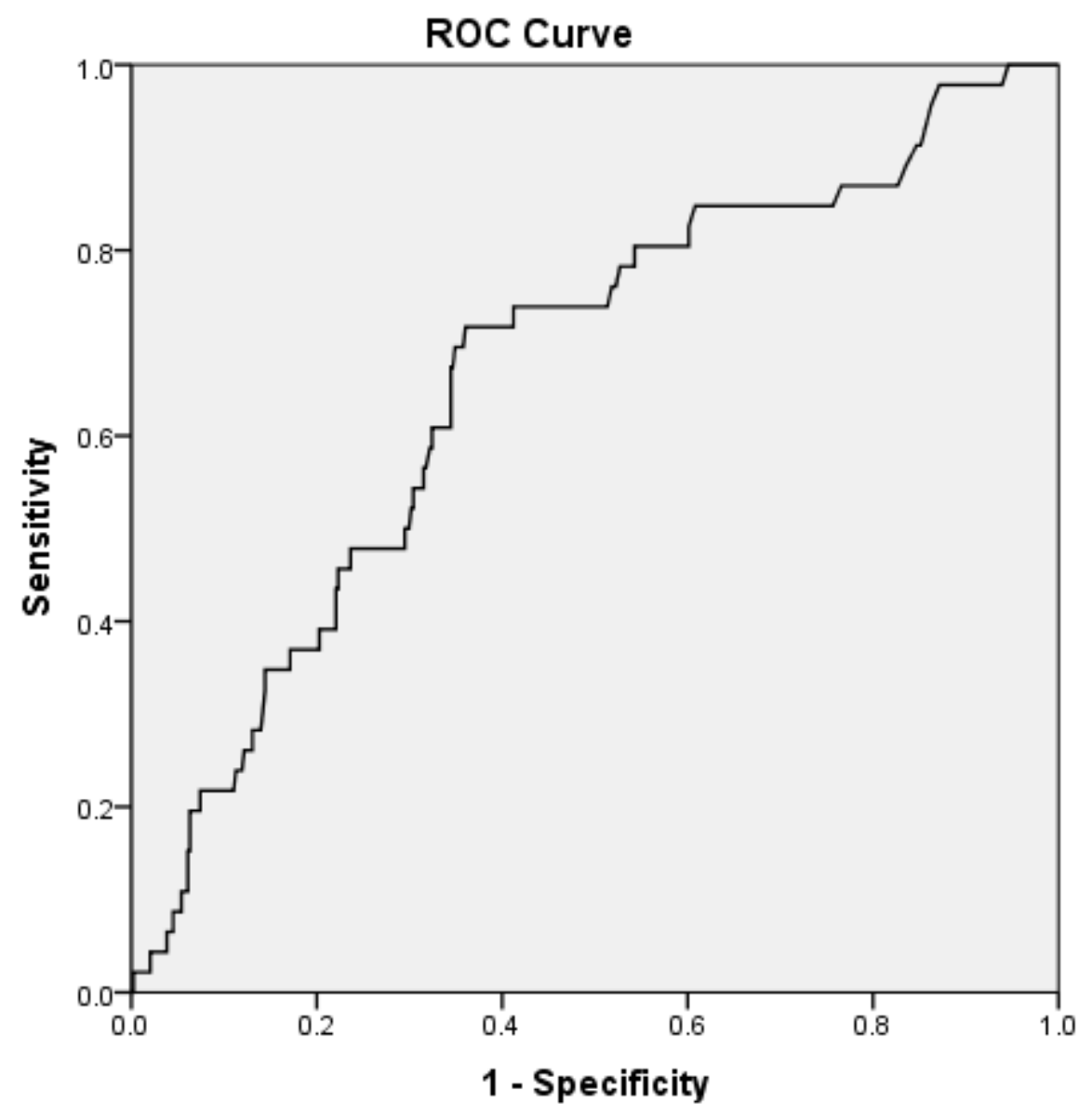

Figure 3

CRP ROC curve Legend: ROC, receiver operating characteristic; NLR, neutrophil to lymphocyte ratio; PLR, platelet to lymphocyte ratio; CRP, C-reactive protein; AUC, area under curve.

\section{Supplementary Files}

This is a list of supplementary files associated with this preprint. Click to download.

- VisualSummaryVladimirOtasevic.pdf 